\title{
多电子原子模型势理论中的矩阵元计算
}

文根旺王簏雅王瑞旦

(湖南师范大学物理系, 长沙 410081)

关语词原子结构、量子化学、势模型、平均值、矩阵元、电子关联

\section{一、引 妄}

最近, 郑能武提出了一个近似描述多电子原子或离子体系中单电子运动的势模型 ${ }^{[1,2]}$. 应 用这一新的势模型, 并根据实验光谱或电离数据确定模型势的参数后 ${ }^{[3]}$, 单电子的径向波函数 可用广义拉盖尔函数表示.

如果以该模型作为量子化学计算的出发点, 我们还需计算各种悬次径向算符的矩阵元. 本 文根据广义拉盖尔多项式的 Taylor 展开及其微分性质, 克服了矩阵元计算时宗量不同的困 难, 导出了一个计算任意幂次算符 $r^{k}$ 的矩阵元与平均值的通式.

\section{二、矩阵元 计算}

使用原子单位制, 可将文献 $[1]$ 的径向波函数方程改写为

$$
\begin{aligned}
& \hat{H}_{l} R_{n l}(r)-E_{n l} R_{n l}(r), \\
& \hat{H}_{l}--\frac{d^{2}}{d r^{2}}-\frac{2}{r} \frac{d}{d r}+\frac{l^{\prime}\left(l^{\prime}+1\right)}{r^{2}}-\frac{2 z^{*}}{r}, \\
& E_{n l}--z_{n}^{*} / n^{* 2} .
\end{aligned}
$$

径向波函数可表示为

$$
R_{m l}(r)-N_{m l}\left(\frac{2 z^{*} r}{n^{*}}\right)^{l *} \exp \left(-\frac{z^{*}}{n^{*}} r\right) L_{n-l-1}^{2 l^{*}+1}\left(\frac{2 z^{*}}{n^{*}} r\right),
$$

其中

$$
\left\{\begin{array}{c}
z^{*}-\sqrt{(z-\sigma)+g \Delta z}, \\
l^{*}-l+d, n^{*}-n+d
\end{array}\right.
$$

为修正的量子数, $N_{n l}$ 为待求的归一化常数. 计算矩阵元 $\left\langle n_{1} l_{1}\left|r^{k}\right| n_{2} l_{2}\right\rangle$ 需要计算以下积分

$$
\left\langle n_{1} l_{1}\left|r^{k}\right| n_{2} l_{2}\right\rangle-\int_{0}^{\infty} r^{k+2} R_{n_{1}, l_{1}}(r) R_{n_{2} l_{2}}(r) d r \text {. }
$$

将波函数(4)式代人(6)式,并令

$$
x=\left(\frac{z_{1}^{*}}{n_{1}^{*}}+\frac{z_{2}^{*}}{n_{2}^{*}}\right) r, a_{1}-\left(2 z_{1}^{*} / n_{1}^{*}\right) /\left(z_{1}^{*} / n_{1}^{*}+z_{2}^{*} / n_{2}^{*}\right),
$$

可得

本文 1989 年 12 月 27 日收到. 


$$
\begin{aligned}
\left\langle n_{1} l_{1}\left|r^{k}\right| n_{2} l_{2}\right\rangle= & N_{n_{1} l_{1}} N_{n_{2} l_{2}} a^{l_{1}}(2-a)^{l_{2}}\left(z_{1}^{*} / n_{1}^{*}+z_{2}^{*} / n_{2}^{*}\right)^{-k-s} \\
& \cdot \int_{0}^{\infty} x^{2} e^{-x} L_{i_{1}}^{i_{1}}(a x) L_{l_{2}}^{n_{2}}((2-a) x) d x
\end{aligned}
$$

其中

$$
\begin{aligned}
& \lambda=l_{1}^{*}+l_{2}^{*}+k+2, \quad s_{1}=n_{1}-l_{1}-1, \quad s_{2}=n_{2}-l_{2}-1, \\
& r_{1}=2 l_{1}^{*}+1, \quad r_{2}=2 l_{2}^{*}+1 .
\end{aligned}
$$

利用 Taylor 展开式:

$$
f(a x)=f[x+(a-1) x]=\sum_{r=0}^{\infty} \frac{(a-1)^{r} \cdot x^{r}}{r !} \cdot \frac{d^{r}}{d x^{r}} f(x)
$$

与广义拉盖尔多项式的微分关系:

$$
\frac{d^{m}}{d x^{m}} L_{n}^{r}(x)-(-1)^{-} L_{n-m}^{r+m}(x), \quad(m \leqslant n)
$$

可得如下展开关系式

$$
L_{n}^{\prime}(a x)=\sum_{m=0}^{n} \frac{(1-a)^{m}}{m !} x^{m} L_{m-m}^{r+m}(x)
$$

因此,(8)式的积分可表示为

$$
\begin{aligned}
& \int_{0}^{\infty} e^{-x} x^{2} L_{s_{1}}^{r_{1}}(a x) L_{s_{2}}^{r_{2}}[(2-a) x] d x \\
& -\sum_{m_{1}=0}^{s_{1}} \sum_{m_{i}=0}^{s_{l}} \int_{0}^{\infty} d x \cdot x^{m_{1}+m_{2}+e^{-x}} e^{-x} \frac{(1-a)^{m_{1}}}{m !_{1}} \frac{(a-1)^{m_{2}}}{m_{2} !} L_{s_{1}^{\prime}-m_{1}}^{r_{1}}(x) L_{s_{2}-m_{2}}^{r_{2}+m_{2}}(x) .
\end{aligned}
$$

恨据积分公式 ${ }^{[4]}$ :

$$
\int_{0}^{\infty} x^{s} e^{-x} L_{n}^{r}(x) L_{n^{\prime}}^{\prime \prime}(x) d x=(-1)^{n+n^{\prime}} \Gamma(s+1) \sum_{k}\left(\begin{array}{c}
s-r \\
n-k
\end{array}\right)\left(\begin{array}{c}
s-r^{\prime} \\
n^{\prime}-k
\end{array}\right)\left(\begin{array}{c}
s+k \\
k
\end{array}\right),
$$

可得积分为

$$
\begin{gathered}
\sum_{=m_{1}=0}^{s_{1}} \sum_{m_{2}=0}^{s_{2}}(-1)^{s_{1}+s_{2}} \frac{(a-1)^{m_{1}}}{m_{1} !} \frac{(1-a)^{m_{2}}}{m_{2} !} \Gamma\left(m_{1}+m_{2}+\lambda+1\right) \\
\cdot \sum_{m}\left(\begin{array}{c}
\lambda+m_{1}-r_{1} \\
s_{1}-m_{1}-m_{3}
\end{array}\right)\left(\begin{array}{c}
\lambda+m_{2}-r_{2} \\
s_{2}-m_{2}-m_{3}
\end{array}\right)\left(\begin{array}{c}
\lambda+m_{1}+m_{2}+m_{3} \\
m_{3}
\end{array}\right),
\end{gathered}
$$

代人(8)式可得

$$
\begin{aligned}
& \left\langle n_{1} l_{1}\left|r^{k}\right| n_{2} l_{2}\right\rangle=N_{n_{1} l_{1}} N_{n_{2} l_{2}}\left(\frac{2 z_{1}^{*}}{n_{1}^{*}}\right)^{l_{i}} \cdot\left(\frac{2 z_{2}^{*}}{n_{2}^{*}}\right)^{l_{2}}\left(\frac{z_{1}^{*}}{n_{1}^{*}}+\frac{z_{2}^{*}}{n_{2}^{*}}\right)^{-l_{i}^{*}-l_{2}^{*}-k^{-2}}(-1)^{n_{1}+l_{1}+m_{2}+l_{2}} \\
& \cdot \sum_{m_{1}=0}^{n_{1}-l_{1}-1} \sum_{m_{2}=0}^{*_{2}-l_{2}-1} \frac{(-1)^{m_{2}}}{m_{1} ! m_{2} !}\left(\frac{z_{1}^{*}}{n_{1}^{*}}-\frac{z_{2}^{*}}{n_{2}^{*}}\right)^{m_{1}+m_{2}}\left(\frac{z_{1}^{*}}{n_{1}^{*}}+\frac{z_{2}^{*}}{n_{2}^{*}}\right)^{-m_{1}-m_{2}} \\
& \times \Gamma\left(l_{1}^{*}+l_{2}^{*}+m_{1}+m_{2}+k+3\right) \sum_{m}\left(\begin{array}{l}
l_{2}^{*}-l_{1}^{*}+k+1+m_{2} \\
n_{1}-l_{1}-1-m_{1}-m_{1}
\end{array}\right)
\end{aligned}
$$




$$
\times\left(\begin{array}{c}
l_{1}^{*}-l_{2}^{*}+k+1+m_{1} \\
n_{2}-l_{2}-1-m_{2}-m_{3}
\end{array}\right)\left(\begin{array}{c}
l_{1}^{*}+l_{2}^{*}+k+2+m_{1}+m_{2}+m_{3} \\
m_{3}
\end{array}\right) .
$$

在(14)式中令 $k-0, n_{1}-n_{2}=n, l_{1}-l_{2}-l$, 于是 $a-1$, 前面两重求和仅有 $m_{1}=0$ 与 $m_{2}=0$ 项, 可求归一化常数 $N_{\mathrm{k} l}$.

$$
\begin{aligned}
\langle n l \mid n l\rangle & -N_{n l}^{2}\left(\frac{2 z^{*}}{n^{*}}\right)^{-3} \Gamma\left(2 l^{*}+3\right) \sum_{m}\left(\begin{array}{c}
1 \\
n-l-1-m
\end{array}\right)^{2}\left(\begin{array}{c}
2 l^{*}+2+m \\
m
\end{array}\right) \\
& -N_{n l}^{2}\left(\frac{2 z^{*}}{n^{*}}\right)^{-3} \Gamma\left(2 l^{*}+3\right) \cdot \frac{2 n^{*}}{n-l-1}\left(\begin{array}{c}
n^{*}+l^{*} \\
n-l-2
\end{array}\right) \\
& -N_{n l}^{2}\left(\frac{2 z^{*}}{n^{*}}\right)^{-3} \Gamma\left(2 l^{*}+3\right) \cdot \frac{2 n^{*} \Gamma\left(n^{*}+l^{*}+1\right)}{(n-l-1) ! \Gamma\left(2 l^{*}+3\right)} \\
& -N_{n l}^{2}\left(\frac{2 z^{*}}{n^{*}}\right)^{-5} \frac{2 n^{*}}{(n-l-1) !} \Gamma\left(n^{*}+l^{*}+1\right),
\end{aligned}
$$

由归一化条件, 即可求出

$$
N_{* l}-\left(\frac{2 z^{*}}{n^{*}}\right)^{3 / 2}\left[\frac{2 n^{*}}{(n-l-1) !} \Gamma\left(n^{*}+l^{*}+1\right)\right]^{-1 / 2} \text {. }
$$

在(15)式中,令 $z^{*}-z, d-0$, 即回到类氢情况, 得到归一化系数为 ${ }^{[3] *}$

$$
N_{n l}-\left(\frac{2 z}{n}\right)^{3 / 2} \sqrt{\frac{(n-l-1) !}{(n+l) ! 2 n}} .
$$

将归一化常数 $N_{m l}$ 代人(14)式, 即得矩阵元与平均值的计算通式:

$$
\begin{aligned}
& \left\langle n_{1} l_{1}\left|r^{k}\right| n_{2} l_{2}\right\rangle \\
& -(-1)^{*_{1}+n_{2}+l_{1}+l_{2}}\left(\frac{2 z_{1}^{*}}{n_{1}^{*}}\right)^{l_{i}}\left(\frac{2 z_{2}^{*}}{n_{2}^{*}}\right)^{l_{2}}\left(\frac{z_{1}^{*}}{n_{1}^{*}}+\frac{z_{2}^{*}}{n_{2}^{*}}\right)^{-l_{i}-l_{i}^{*}-k-s} \\
& \cdot\left[\frac{n_{1}^{* 4}}{4 z_{1}^{* 3}} \frac{\Gamma\left(n_{1}^{*}+l_{1}^{*}+1\right)}{\left(n_{1}-l_{1}-1\right) !}\right]^{-1 / 2}\left[\frac{n_{2}^{* 4}}{4 z_{2}^{* *}} \frac{\Gamma\left(n_{2}^{*}+l_{2}^{*}+1\right)}{\left(n_{2}-\cdots l_{2}-1\right) !}\right]^{-1 / 2} \\
& \text { - } \sum_{m, 1=0}^{n,-l_{1}-1} \sum_{m=0}^{n,-l_{1}-1} \frac{(-1)^{-m_{2}}}{m_{1} ! m_{2} !}\left(\frac{z_{1}^{*}}{n_{1}^{*}}-\frac{z_{2}^{*}}{n_{2}^{*}}\right)^{m_{1}+m_{2}}\left(\frac{z_{1}^{*}}{n_{1}^{*}}+\frac{z_{2}^{*}}{n_{2}^{*}}\right)^{-m_{1}-m_{2}} \\
& \text { - } \Gamma\left(l_{1}^{*}+l_{2}^{*}+m_{1}+m_{2}+k+3\right) \sum_{m_{1}=1}^{s}\left(\begin{array}{l}
l_{2}^{*}-l_{1}^{*}+k+m_{2}+1 \\
n_{2}-l_{1}-1-m_{1}-m_{3}
\end{array}\right) \\
& \text { - }\left(\begin{array}{c}
l_{1}^{*}-l_{2}^{*}+k+m_{1}+1 \\
n_{2}-l_{2}-1-m_{2}-m_{3}
\end{array}\right)\left(\begin{array}{c}
l_{1}^{*}+l_{2}^{*}+k+2+m_{1}+m_{2}+m_{3} \\
m_{3}
\end{array}\right) \text {, }
\end{aligned}
$$

其中 $S-\min \left\{n_{1}-l_{1}-1-m_{1}, n_{2}-l_{2}-1-m_{2}\right\}$, 并由积分收玫条件得 $k>-l_{1}^{*}-l_{2}^{*}-3$.

将(17)式中的 $n_{1} l_{1}$ 与 $n_{2} l_{2}$ 的位置对换, 表达式的值不变, 即矩阵元 $\left\langle n_{1} l_{1}\left|r^{k}\right| n_{2} l_{2}\right\rangle$ 关于 初未量子态是对称的,这符合厄米矩阵元对称性的要求。

- 本文与文献 $[1,2]$ 相一致,所采用的 $j^{2}$ 义拉盖尔函数与文献 [4]相同, 不同于昷子力学教科书上流行的记号,两者的负

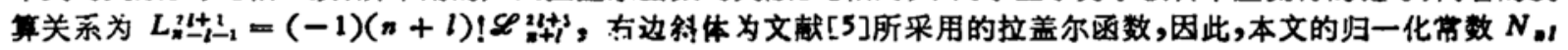
与文嗝[5]相差一个因子 $(n+l) !^{-1}$. 
(17)式包含了平均值的计算式, 只需令 $n_{1}=n_{2}=n, l_{1}=l_{2}=l$, 这时, 对 $m_{1}$ 与 $m_{2}$ 求 和的各项中只有 $m_{1}$ 与 $m_{2}$ 都为零的这项不消失, 于是可得出平均值的计算式为

$$
\begin{aligned}
&\left\langle n l\left|r^{k}\right| n l\right\rangle-\left(\frac{n^{*}}{2 z^{*}}\right)^{k} \frac{(n-l-1) !}{2 n^{*}} \frac{\Gamma\left(2 l^{*}+k+3\right)}{\Gamma\left(n^{*}+l^{*}+1\right)} \\
& \cdot \sum_{m=s^{\prime}}^{*-l-1}\left(\begin{array}{c}
k+1 \\
n-l-1-m
\end{array}\right)^{2}\left(\begin{array}{c}
2 l^{*}+k+2+m \\
m
\end{array}\right),
\end{aligned}
$$

其中求和限为二项式系数的非零范围决定, 因此, $S^{\prime}-\max \{0, n-l-1-k-1\}$.

从(18)式过渡到类氢原子的计算式, 只需要令各量子亏损为零, 即 $z^{*}=z, l^{*}-l, n^{*}=$ $n$, 可导出与 Bockasten ${ }^{(6)}$ 相同的计算式,这说明本文推导的公式是正确的.

\section{三、结 论}

本文根据广义拉盖尔函数的性质, 导出了多电子原子模型势理论中电子径向幂算符的矩 阵元与平均值计算的通式. 使用本文的公式, 我们可从郑能武的模型势方法所建立的解析径 向波函数作为多电子原子或离子径向波函数的零级近似，在此基础上进行多重态计算和组态 相互作用计算, 从而可得出精确描述多电子原子运动规律的波函数与能谱以及各类跃迁振子 强度,进而可进行各种物理力学过程和量子化学的研究.

\section{考文献}

[1] 郑能武,科学通报, 30(1985),23: 1801 .

[2] 郑能武,科学通报, 31(1986),17: 136 .

[3] 郑能武,科学通报, 32(1987),5: 354 .

[4] 王竹涺、部效仁,待殊函数概论,科学出版社, 1979,364.

[5] Landau, L. D. and Lifshitz, E. M., Quantum Mechinics, Pergamon Press, 1976, 117.

[6] Bockasten, K., Phys. Rev., A9(1974), 3: 1087-1089. 